\title{
El Comportamiento Humano
}

\section{Carlos Eduardo Cobo*}

- Candidato a Doctor en Dirección de Empresas de la Universidad de Salamanca, España. Magíster en Administración de Empresas, Especialista en Administración de Empresas e Ingeniero Electricista de la Universidad del Valle, Colombia. Actualmente es profesor de tiempo completo de la Universidad del Valle. 


\section{INTRODUCCIÓN.}

Hace un año aproximadamente me interese en estudiar las causas del comportamiento humano, ya que soy un convencido de que la fuerza básica que mueve cualquier empresa nace del ser mismo cuando se dan ciertas condiciones intrínsecas y extrínsecas a él. El problema del comportamiento humano ha tenido diferentes soluciones a través de la historia, desde antes de que se iniciaran las investigaciones psicológicas, filósofos, antropólogos y teólogos desarrollaron constructos acerca del porque del comportamiento de una persona en una determinada situación.

Este articulo pretende hacer una síntesis incompleta de las diferentes teorfas o paradigmas que han intentado resolver el problema.

He agrupado las Teorías que explican las actitudes y los comportamientos humanos en 3 categorías buscando facilitar al lector la comprensión de los elementos comunes y diferentes entre los modelos.

0 El comportamiento organizacional

1 Teorías cognoscitivas

2 Teorias psicoanalíticas

3 Teorías conductistas

5 Síntesis

\section{EL COMPORTAMIENTO ORGANIZACIONAL.}

El Comportamiento organizacional (C. O.) es un área del conocimiento apli- cado, que estudia la manera como los individuos y grupos actúan dentro de aas organizaciones, con el propósito de elaborar algunos principios que permitan entender el fenómeno humano en las organizaciones. J. Clifton Williams define el C. O. como «el estudio del comportamiento individual y grupal dentro de los sistemas organizacionales, mediante el análisis de sus contingencias y la comprensión de sus procesos, utilizando conocimientos derivados de la sociología, la psicología, la economía y la antropología. Estos conocimientos se integran sistemáticamente y contribuyen al logro de la efectividad y del desarrollo humano y organizacional para su continuidad y supervivencia.» (Williams, 1984). Otra definición de C. O. es la dada por J. Kelly en donde el C.O. «es el estudio de los organizadores, quiénes son, qué hacen, con quién se comunican y cómo lo hacen; brevemente, su enfoque es el campo de las interacciones entre los hombres de la organización, pero es también el estudio de los organizados y de cómo se organizan para organizar a los organizadores. Inherente a este enfoque es la noción de reciprocidad que asume que todas las acciones sociales invitan, inevitablemente, a reacciones; y el hecho de que muchas de ellas no se pueden anticipar es lo que confiere a la vida lo bizarro de lo inesperado y es lo que hace a la vida organizacional tan fascinante» (Williams, 1984).

El comportamiento organizacional al estudiar las diferentes conductas de 
las personas tiene en cuenta el clima organizacional donde ellas desempeñan su trabajo diario. El conocimiento generado por esta área de estudio es utilizado para: 1. mejorar la efectividad de las organizaciones respetando el orgullo y la dignidad humana y 2 . busca promover un desarrollo humano integral. (Davis, 1988). El comportamiento humano, de acuerdo a la Psicología, son todas las actividades expresadas fisicamente por el ser humano y todos sus procesos mentales manifestados por medio de expresiones orales como los sentimientos y los pensamientos, que un individuo manifiesta cuando se encuentra en una situación social en particular. Casi todos nuestros comportamientos conscientes son motivados, es decir que buscan satisfacer un deseo, el cual es la manifestación sentida de una necesidad. Nuestras actitudes son conductas, conscientes o inconscientes, que emprendemos como respuesta al estímulo percibido y que proviene de otro ser vivo, de algún fenómeno natural o de algún objeto en particular. (Schein, 1982).

\section{LAS ACTITUDES}

Cada uno de nosotros tenemos actitudes hacia casi todas las cosas o personas del mundo en las que hayamos pensado en algún momento. Las actitudes son las formas de reaccionar (conductas, comportamientos) a algún estímulo producido por un ser vivo o por alguna otra cosa; cuando la respuesta la hacemos verbalmente se de- nomina opinión. Las actitudes se componen de tres elementos: el cognitivo, el emocional y el conductal. Miremos un ejemplo, Usted lee un artículo en un periódico sobre el aborto, su actitud frente a la idea se descompone en 1. lo que piensa: creo que es antinatural; 2 . lo que siente: tristeza por la cantidad de niños que mueren; 3 . su conducta: no aceptar que su compañera aborte. Las actitudes corresponden a las expresiones psíquicas del ser humano: el pensar, el sentir y la voluntad (Monroy, 1994). Los tres componentes de las actitudes frecuentemente se encuentran en contradicción unos con otros: Se piensa que es malo fumar pero se actúa fumando. Siente repulsion hacia el superior pero lo halaga. $\mathrm{Al}$ ser las actitudes conductas, pueden ser desarrolladas o modificadas. Todos nosotros podemos recordar experiencias en donde alguien, papá, mamá, maestro, el médico o el sacerdote, ha tratado de convencernos de que cambiemos algún hábito, actitud o conducta respecto a algo y algunas veces han logrado el desarrollo o el cambio de un comportamiento. Existen tres elementos que aparecen cuando hay un intento de cambio de conducta: 1 . La fuente que induce el cambio, 2. las características del medio usado para inducir el cambio y 3 . las características de las personas a las cuales se quiere inducir al cambio. Cuando hablamos de la fuente que induce al cambio nos referimos a las características de las personas que desean que nosotros cambiemos una 
actitud, se ha descubierto que los cambios se suceden con mayor facilidad, si nosotros confiamos en los conocimientos, la experiencia y la buena voluntad para con nosotros de quien nos esta tratando de cambiar. La fuente puede utilizar muchos medios para inducir los cambios desde charlas personales hasta videos pasando por los mensajes escritos. Los mensajes llevados por estos medios se pueden dirigir hacia nuestro raciocinio o hacia nuestros sentimientos según lo considere fuente. Una estrategia muy usada con los públicos es recurrrir a mensajes que infunden algo de miedo a quien lo recibe. Finalmente están las personas quienes reciben el mensaje de cambiar, lo interpretan según su individualidad y actúa ya sea reafirmándose en su actitud o comprometiéndose con el cambio.

\section{TEORIAS QUE EXPLICAN LAS ACTITUDES Y COMPOR- TAMIENTOS}

Existen varias teorías que explican la formación o modificación de las actitudes las cuales voy a agrupar en tres grandes grupos: las teorías cognoscitivas, las teorías Conductistas y las teorías Psicoanalíticas.

\section{Teorías cognoscitivas}

Tienen su fundamento básico en la visión de que son las necesidades internas del individuo que provocan un determinado comportamiento o ac- titud. Consideran que todos nuestros actos son dependientes de nuestras propias acciones y son inherentes a la vida de cada uno de nosotros. Los cognitivistas no ven las experiencias de la vida como la causa básica de nuestras actitudes, para ellos las causas hay que buscarlas en las necesidades de cada individuo. Para ellos el individuo es el actor, el hacedor, el que construye su propio mundo de necesidades y desarrolla consecuentemente sus actitudes y comportamientos para satisfacer sus deseos. Dentro de esta categoría ubicamos los siguientes modelos:

\section{Teoría de la motivación de McGre-} gor

Esta teoría se fundamenta en la idea de que las actitudes y los comportamientos son motivados por las necesidades de cäda ser humano, las cuales van cambiando de acuerdo a la situación espacio-temporal que enfrenta el individuo. Otro concepto es el de que cada vez que una necesidad es satisfecha, el individuo buscara otra necesidad que satisfacer, en un proceso que solo termina con la muerte del ser. En este modelo las necesidades humanas son ordenadas ascendentemente de la siguiente manera: En el nivel más bajo se encuentran las necesidades vitales, salud, alimentación, abrigo y sexo, luego vienen las necesidades de estabilidad como protección a las amenazas del entomo, la búsqueda de estabilidad o el aseguramiento 
de la satisfacción de las necesidades vitales cuando este incapacitado para trabajar; Después están las necesidades sociales que son el reconocimiento, la aceptación, el amor, la amistad y el afecto que el ser humano encuentra en los distintos grupos a los cuales pertenece. La siguiente es la necesidad del ego que son las relacionadas consigo mismo, como auto confianza, autoconocimiento o autoestima y las relacionadas con la reputación social, como posición social o respeto de los demás. En el nivel más alto de la jerarquía de necesidades encontramos las de autorrealización que involucra el deseo que tenemos cada uno de nosotros de ser cada vez más de lo que somos y el deseo de maximizar nuestro potencial intelectual y cultural. La principal critica a este modelo es la dificultad para validar científicamente el modelo debido al manejo cualitativo de las variables. (Chiavenato, 1981).

\section{Teoría de la auto actualización.}

Abraham H. Maslow (1908-1970) se dedicó al estudio de las personas sanas, examinó la alegría, el entusiasmo, el amor y la satisfacción. Construyó su teoría reconociendo que todo ser humano tiene dos tipos de necesidades: las primarias que corrigen deficiencias y las secundarias que persiguen el nivel más alto de existencia del ser humano. Tan pronto se satisfagan las necesidades primarias, el ser se comienza a preocupar por las necesidades de otro nivel superior que corres- ponden a aquellas que nos dejan gratificaciones de tipo espiritual o psíquico. La teoría presenta en cinco niveles la jerarquía de las necesidades: 1 . necesidades físicas básicas, 2 . necesidades de protección y seguridad, 3. necesidades de pertenencia y sociales, 4 . necesidades de estima y estatus y 5 . necesidades de actualización de sí mismo. Los dos primeros niveles se refieren a la sobrevivencia del individuo y mientras estas necesidades no sean satisfechas, ninguna otra necesidad de orden superior se hará presente. Los críticos de Maslow invalidan su teoría porque ha sido muy dificil validarla en investigaciones posteriores, sin embargo le dan valor en el sentido de que es un marco filosófico que permite describir las actitudes de las personas dentro de una organización. (Gibson, 1984)

\section{Teoría centrada en la persona}

Carl Rogers (1902-197) ha construído un modelo sobre el comportamiento humano que añade a los factores factores psicológicos observables, experiencias humanas únicas como son: el amor, el odio, la alegría, la tristeza, el sentido de vida, la responsabilidad, el orgullo y el miedo, entre otras. La principal idea sobre la que se edifica esta teoría es la de que todos los seres humanos necesitamos encontrar nuestro «yo» real para aceptarnos y valoramos por lo que somos. El proceso de búsqueda del «yo» es continuo, activo y dinámico, guiado por una 
tendencia a la actualización que poseen todos los organismos vivos, la cual puede cambiar de sentido pero no abandona al ser vivo sino hasta su muerte. Todas las actitudes de un ser normal siempre estarán guiadas hacia su crecimiento y reproducción, sin tener en cuenta si su entorno genera estímulos favorable o desfavorables. La satisfacción, dentro de esta teoría, se da si hay acercamiento entre lo que nos gustaría ser y lo que somos, entre lo que pensamos acerca de algo y lo que creo que los demás piensan de ese algo, entre lo que pienso, siento y hago. (Papalia, 1987).

\section{Teoría Motivación-Higiene de Herzberg.}

Dentro de este modelo existen dos factores que influyen separadamente en el comportamiento de un individuo: los factores higiénicos y los factores motivacionales. Los primeros, si están presentes no producen motivación en el individuo, pero si están ausentes producen insatisfacción en la labor que esté desempeñando, los factores higiénicos están relacionados con el medio ambiente o contexto donde se desarrolla el trabajo, algunos de ellos son: salario, seguridad en el empleo, condiciones en el trabajo, la vida personal, etc. Los factores motivacionales, si están presentes producen satisfacción en el trabajo y si no están presentes producen no satisfacción; estos factores están relacionados con el contenido del trabajo y algunos de ellos son: Reconocimiento por los objetivos logrados, trabajo interesante, posibilidad de desarrollo, asignación creciente de responsabilidades, etc. El comportamiento de una persona está determinado por el juego de las intensidades de ambos factores. Los críticos del modelo rechazan la idea de los dos factores independientes de motivación, sosteniendo que no puede existir sino un sólo factor a través de un continuo. Otra crítica es la dificultad para validar el modelo. (Gibson, 1984).

Teoría de las necesidades de logro, de afiliación y de poder.

David C. Mc Clelland encontró en su investigación que existen cuatro patrones de motivación que determinan las actitudes de las personas durante su vida, estos patrones son producto del sistema cultural en que ha vivido, principalmente durante su infancia. Los patrones son: 1. La Necesidad de logro que produce actitudes que buscan superar cualquier obstáculo que le impida alcanzar una meta pre-establecida por el individuo y que generalmente es difícil de lograr. 2. La Necesidad de afiliación que origina actitudes que le permiten relacionarse socialmente con otros individuos. Las personas de este tipo de patrón, les gusta obtener reconocimiento por sus actitudes positivas hacia los demás y por su colaboración desinteresada, además valoran mucho la amistad. 3 . La necesidad de poder genera un deseo de influir en otros y modificar sus 
situaciones. Actitudes más comunes entre las personas con este tipo de patrón son de dominio, de conquista, de control, de lealtad. 4. La Necesidad de competencia produce actitudes dirigidas a conseguir la excelencia y la alta calidad en todas las actividades que emprende. Las personas con este patrón son compulsivas por la eficiencia en los procesos que llevan al logro de objetivos y se impacientan con sus colaboradores cuando ellos no logran altos niveles de productividad. (Chiavenato, 1981).

\section{Teoría de campo de Lewin}

Esta teoría se fundamenta en la siguiente idea: el comportamiento de los seres humanos no depende ni del pasado, ni del futuro sino del espacio psicológico real y actual, donde se desenvuelve la vida del individuo, el cual está relacionado con las necesidades del mismo. Cada uno de nosotros asigna de acuerdo a sus experiencias, conocimientos y percepciones, unas "valencias» a los objetos, personas o situaciones, las cuales pueden ser positivas si prometen satisfacer necesidades presentes de la persona o pueden ser negativas si prometen generar algún daño al individuo. Un objeto, persona o situación de vaIencia positiva produce una fuerza que atrae al individuo, por el contrario un objeto, persona o situación de valencia negativa produce una fuerza que repele al individuo. De la suma vecto- rial de estas fuerzas, que la persona percibe en determinado momento, se genera una fuerza resultante que induce una locomoción en cierta dirección. El comportamiento del individuo está dado por la dírección de la fuerza resultante y puede ser de repulsión del objeto, persona o situación o puede ser de atracción. (Chiavenato, 1981).

\section{Teoría de las Expectativas}

Victor Vroom tomó algunos conceptos planteados por $\mathrm{K}$. Lewin y por E. Tolman y desarrolló su modelo en donde la motivación y el comportamiento de un individuo se puede determinar en función de sus expectativas y sus valencias. Las expectativas son creencias que un individuo tiene acerca de comportamientos específicos para lograr algún resultado. Para Vroom existen dos clases de expectativas: la de esfuerzo- desempeño y la de đesempeño-resultado, la primera se refiere al grado de convicción que tiene el empleado de que una determinada acción en el trabajo permitirá lograr el objetivo deseado, es el caso del estudiante que cree que entre más tiempo dedique a realizar su tarea logrará terminarla a tiempo; la otra expectativa es la de desempeño-resultado la cual se refiere al nivel de convencimiento que el individuo tiene de que recibirá una recompensa por la tarea cumplida, en el caso del estudiante sería el grado de certeza de que sacará una buena nota en el trabajo. La valencia, como vimos en la teoría de campo de Lewin, 
se refiere al valor que una persona asigna a determinado objeto, persona o situación en determinado momento de la vida. En el modelo, la motivación que induce un determinado comportamiento $o$ actitud depende del producto de los siguientes tres factores: la valencia, la expectativa esfuerzo-desempeño y la expectativa desempeño- resultado. El procedimiento para califiacr las variables es el siguiente: la valencia hacia un objeto, situación o persona varia entre +1 y -1 , donde +1 corresponde al máximo valor asignado a una valencia positiva y -1 es el máximo valor asignado a una valencia negativa. La calificación de la valencia es subjetiva por parte del individuo y depende del momento en que se califique, por ejemplo a una rosquilla de pan una persona le pude dar una valencia de 0.8 si se le cuestiona por ella antes de almorzar, pero si se le pregunta después de que ha satisfecho su necesidad de hambre, la misma rosquilla le asignaran un valor de 0.2. Las expectativas se miden con el concepto de probabilidad, por lo tanto sólo puede tomar valores entre 0 y 1 . Cuando medimos la expectativa esfuerzo-desempeño se le pide al individuo que evalúe la posibilidad de éxito de la estrategia que va a usar para alcanzar el objetivo y en la expectativa de desempeño resultado se le pide a la persona la probabilidad que el cree tener de alcanzar una recompensa si logra el objetivo previsto. La principal crítica que se le hace a esta teoría es que no ha sido validada científicamente, ya que existen tantas causas y efectos en una misma situación, que es muy difícil para una persona percibir y calificar cada uno de los tres factores con precisión, es decir que la evaluación de los factores no es fácil medirlos científicamente. Otra crítica esta orientada hacia la ausencia en ei modelo de otros factores que permitan explicar mejor las actitudes del empleado hacia un objeto, situación o persona, como por ejemplo el compromiso del empleado, el clima organizacional, el estilo de dirección, etc. (Davis, 1988).

\section{Teoría de la fijación de objetivos para el desempeño}

Para Edwin Locke el comportamiento de las personas dentro de una organización está determinado por cuatro factores 1. Los objetivos fijados por la organización a cada trabajador, en cuanto a su grado de claridad, de especificidad y de interes que despierta en el empleado, 2. Las características que presenta la organización en relación a la tecnología, su estructura, el liderazgo y el clima de trabajo, 3. Ia personalidad del empleado en cuanto a sus valores, sus intereses y objetivos personales, el grado de escolaridad, la edad y su estabilidad emocional, 4. la nivel de certeza que percibe el empleado en cuanto a la obtención de recompensas si cumple con los objetivos asignados por la organización. (Blank, 1990). 


\section{La teoría de las atribuciones de $\mathrm{F}$. Heider.}

Esta teoría busca explicar las causas del comportamiento de las personas en función de dos factores: las caracteristicas personales de la persona y las circunstancias del ambiente donde se encuentra el individuo, cada uno de los factores debe recibir una calificación binaria de estabilidad: inestable o estable. De acuerdo a la combinación de ambas evaluaciones resul$\tan$ cuatro posibles explicaciones de la actitud exitosa o el fracaso del empleado en el desempeño de una tarea, en lo personal: Su habilidad o su esfuerzo, y en lo circunstancial: la dificultad de la tarea o la suerte. Este modelo debe integrarse a otros teorías sobre el comportamiento para aumentar su utilidad.

\section{Teorías Psicoanalíticas.}

Se fundamentan en la historia pasada del individuo que marca su inconsciente de una manera particular, $y$ este a su vez genera necesidades que impulsan una conducta determinada segun las circunstancias. Las marcas resultan del constante conflicto entre los instintos naturales y las restricciones que la sociedad impone sobre cada uno de nosotros. La naturaleza de estos conflictos depende de la etapa de desarrollo en que se encuentre el individuo en un momento determinado. Dentro de esta categoría encontramos los siguientes enfoques:

\section{La escuela Psicoanalítica} Freudiana

Sigmund Freud( 1856-1939) a ravés de la observación clínica de pacientes que manifestaban un comportamiento anormal, construyo un paradigma que presentaba los impulsos biológicos inconscientes de naturaleza sexual, como el principal factor de influencia sobre la conducta humana. Su principal discípulo fue Erik Erikson y otros como Carl Jung, Alfred Alder y Karen Horney, han construido nuevos enfoques modificando las ideas básicas freudianas.

Para Freud los seres humanos pasamos diferentes etapas de acuerdo a nuestro desarrollo psicosexual, las cuales bautizó según la parte del cuerpo que se constituye como fundamental para la satisfacción del ser. Las etapas se van agotando en una forma secuencial y el tiempo de duración de cada una de ellas depende del nivel de maduración propio de cada ser. Las etapas son: La etapa Oral (desde el nacimiento hasta los 18 meses), en la cual el niroo recibe gratificación a través de la boca y chupar es muy importante. La etapa Anal (de los 18 meses hasta los 3 años), en la cual el nino recibe gratificación en el ano durante la defecación. La etapa Fálica (desde los 3 años hasta los 5 años), en la cual la estimulación genital es la parte del cuerpo que genera mayor satisfacción. La etapa de Lactancia, en la cual el nino se encuentra en una época de calma sexual relativa, sin que anule la sexua- 
lidad, ya que a esta edad, entre los $6 \mathrm{y}$ los 12 años, los ninos continúan explorando sus zonas genitales, explorándose entre unos y otros, y pensando y hablando de temas sexuales. La etapa Genital, en la cual los cambios hormonales dan paso a la sexualidad adulta madura. Los impulsos sexuales se orientan hacia personas de fuera de la familia y generalmente del sexo opuesto. Las actitudes y las conductas que observamos en la vida adulta son determinadas por las experiencias que ocurrieron durante cada una de estas etapas. El exceso o la ausencia de satisfacción en una etapa puede «marcar» (proceso de fijación) al individuo a esa etapa en particular. Fijación en términos Freudianos significa que el individuo permanece con un lazo de inmadurez ligado a una eta$\mathrm{pa}$, lo cual le puede generar obstáculos para alcanzar su desarrollo normal. Freud basándose en observaciones de la conducta de los individuos y en el análisis de las expresiones y sentimientos de sus pacientes, planteó la existencia de tres fuerzas, no físicas, presentes en cada uno de nosotros, las cuales aparecen con distintas intensidades en cada una de las etapas de su teoría. Estas fuerzas las denominó: 1. él «id», fuente inconsciente de motivos y deseos que exige la gratificación inmediata, opera bajo el principio del placer. Él «id» esta compuesto por las necesidades básicas tanto de vida (instintos de vida) como de destrucción (instintos de muerte), estos instintos son la base de todo comportamiento humano durante toda la vida. 2. El «ego», opera bajo el principio de la realidad, es desarrollada por el niño a partir del momento en que se da cuenta de que todo lo que desea no es posible conseguirlo automáticamente y por lo tanto debe «idear» una estrategia para alcanzar lo deseado. 3. El «súper ego"), se desarrolla a partir de los 5 años de edad y opera bajo el principio de perfección. Esta constituido por todos los valores que tanto padres, maestros, amigos y en general la sociedad, transmiten al niño como ideales. Es el «súper yo» el encargado de interiorizar en el niño lo que es bueno y lo que es malo. Las tres fuerzas deben permanecer idealmente en equilibrio, permitiendo aue el individuo tenga la espontaneidad del «ello», la racionalidad del «yo» y la moral del «súper yo». Cuando ocurre un desequiliorio de fuerzas se genera una ansiedad que es eliminada por medio de los mecanismos llamados de defensa: El desplazamiento, la sublimación, la represión, la regresión y la proyección, entre otros. Freud insistio en que la conducta del individuo está muy determinada por io que ocurra en las tres primeras etapas. Miremos algunos ejemplos: una persona fijada con la etapa oral puede, en la etapa adulta, desarrollar una conducta ambiciosa y dependiente, mientras que otra persona fijada a la etapa anal llegue a comportarse en forma avara, destructiva, cruel y obstinada. El gran aporte de Freud consiste en haber introducido el concepto de «inconsciente» como 
el conjunto de ideas y sentımientos que dirigen nuestros pensamientos y nuestras acciones, generando comportamientos anormales cuando se presentan mecanismos de defensa permanentes. La principal crítica se basa en el hecho de que Freud sólo consideró los instintos sexuales básicos en los seres humanos. (Papalia, 1985).

\section{Teoría de Erikson sobre el desa- rrollo Psicosocial.}

Erikson trabajó el concepto Freudiano del «ego» interesándose en la influencia que tiene la sociedad sobre la personalidad y el comportamiento de los individuos. Consideró que durante toda la vida de un ser humano se presentaban 8 situaciones de crisis y de la manera en que se resuelva o no, dependen las actitudes y conductas del individuo. A diferencia de Freud, quien centra su teoría en los factores biológicos, Erikson da crédito a los factores sociales y culturales en su teoría. Las crisis son: 1 . Confianza básica contra desconfianza básica (del nacimiento a los 18 meses). Cuando al niño se le da un cuidado constante que satisfaga sus necesidades básicas y lo haga sentir seguro y confortable, éste podrá resolver con éxito esta crisis, lo cual le permitirá al individuo valorar a los demás y determinar en quien confiar y en quien no confiar. 2 . Autonomía contra vergüenza y duda (de los 18 meses a los 3 años). En esta etapa se debe proveer al niño de un adecuado nivel de control, de tal ma- nera que se le permita explorar su entorno permitiéndole conocer hasta donde llega su autonomía y cuales son sus limitaciones. 3. Iniciativa contra Culpa (de los 3 años a los 6 años). Se debe promover al niño para que aprenda a regular su poder que impulsa iniciativas y a determinar su responsabilidad por acciones incorrectas, sin que el sentido de culpa sea excesivo. 4 . Laboriosidad contra inferioridad (de los 6 a los 12 años). De esta crisis el nino debe salir manejando los conceptos de productividad y competencia de acuerdo a los conocimientos del nirio. Se debe evitar que el niño se sienta inferior frente a sus compañeros. 5 . Identidad contra la confusión de roles (Adolescencia). Durante esta época la tarea más importante del individuo es resolver la pregunta «quien soy yo?». En esta época suceden cambios fisiológicos fuertes que lo pueden confundir respecto a lo que será su futuro. Las relaciones con jóvenes de su misma edad permitirá conocerse mejor, ya que a través de compartir sus sentimientos y pensamientos, logrará perfilar su propia identidad. 6 . Intimidad contra aislamiento (Adulto joven). La lucha por no perder su «ego» frente al deseo de compartir una relación más intima con otro individuo es la característica de esta crisis. Un exceso de aislamiento que busque mantener su propia individualidad puede llevar a la persona a un estado de soledad, y por el contrario una entrega «total» a otro individuo puede arriesgarlo a una pérdida de identidad. 7. Generatividad 
contra estancamiento (madurez). Después de los 40 años el individuo demuestra mucho interés por guiar y enseñar a la siguiente generación de individuos, pasando su propia experiencia a otros individuos más jóvenes. Cierto grado de estancamiento para dar a la creatividad cierto reposo es bueno, sin llegar a excesos que lleven al individuo a la autoindulgencia. 8. Integridad del YO contra la desesperación (senectud). Las personas mayores deben aprender a aceptar la vida que se ha llevado, sin remordimientos por lo que podían haber hecho y no hicieron. La persona que no puede aceptar la forma como ha vivido puede caer en el desespero al darse cuenta que ya no hay tiempo para rehacer las cosas.

Según este enfoque, todos los seres nos vemos enfrentados a resolver en forma equilibrada cada una de las crisis, de tal manera que nuestros comportamientos sean lo más «normales» dentro del contexto cultural y la época en que se viva. (Papalia, 1987).

\section{La escuela Kleniana}

Para Melaine Klein el comportamiemto humano es el resultado de la definición del conflicto primario entre las buenas y las malas experiencias tempranas entre el niño y el pecho de su madre o su sustituto. La lucha da al niño referencias que le permiten estructurar un modelo inconsciente de conducta y de sentimientos que per- dura en su vida. En el desarrollo del infante se presentan dos etapas fundamentales según el modelo de Klein: 1. la fase persecutoria, en donde el niño desarrolla estrategias de defensa contra la angustia de perder el pecho de la madre, lo que lo llevaría a la muerte; de otro lado, desarrolla elementos de afirmación e integración a la vida y sentimientos de amor y felicidad cuando el pecho le suministra una alimentación placentera. En esta fase existen en forma independiente el pecho "malo» y el pecho «bueno». La otra fase se denomina la fase depresiva, en donde el niño se da cuenta que el buen y el mal pecho son uno mismo, y que él ha estado amando y odiando la misma cosa. (Morgan, 1991).

\section{La teoría Conductista.}

Partiendo de estudios de laboratorio sobre el condicionamiento e influenciados notablemente por el pensamiento positivista de la época, John B Watson (1878-1958) y luego B.F. Skinner (1904- ) utilizaron el método científico para estudiar el comportamiento con animales como una forma de aprender más sobre los seres humanos. El rechazo a considerar cualquier factor psicológico no observable y su negación a aceptar los procesos cognitivos son los puntos más débiles de la teoría conductista. Estas teorías presentan la conducta como una consecuencia externa al individuo. Dentro de esta categoría se encuentra: 


\section{La teoría del condicionamiento Clásico de I. Pavlov}

En el condicionamiento clásico $€ 1$ animal o la persona aprende a responder a un estímulo previamente neutro, cuando este es asociado repetidamente con un estímulo incondicionado. El condicionamiento clásico tiene tres fases: 1. Fase anterior al condicionamiento en la cual el estímulo incondicionado (la carne en el caso de los perros de Pavlov) produce la respuesta incondicionada automáticamente (la salivación del perro). El estímulo neutro (la campana) no produce salivación. 2. Fase del condicionamiento en la cual el estímulo incondicionado (la carne) es asociado con el estímulo neutro (la campana). 3. Fase después del condicionamiento en la cual el estímulo neutro (la campana) es ahora el estímulo condicionado y produce una respuesta condicionada (la salivación), la cual es similar a la respuesta incondicionada producida inicialmente. Este proceso que acabamos de presentar es el de adquisición de una respuesta condicionada y de la misma forma podemos crear procesos para extinguir o recuperar las respuestas condicionadas. El condicionamiento clásico ha sido utilizado muy frecuentemente en el mundo de los negocios, por ejemplo el caso del vendedor que invita a su cliente potencial a cenar, lo que está haciendo es desarrollar en el cliente una respuesta condicionada, en donde se asocian la propuesta del vendedor con los buenos sentimien- tos generados por una agradable comida. En síntesis todos las personas están condicionadas por muchos aspectos del entorno, y es común que las organizaciones utilicen el condicionamiento clásico para promover actitudes y conductas deseadas por la empresa. Para Jhon B. Watson, denominado el padre del conductismo, el hogar es el laboratorio donde se condicionan los niños. Es duramente criticado por someter a bebes a experiencias para probar que una reacción emocional condicionada podía persistir por periodos de tiempo superiores a un mes.

Experimentos de este tipo dieron origen al código de ética de la asociación psicológica americana, la cual no permite desarrollar experiencias del tipo mencionado. (Papalia, 1987)

\section{La teoría del condicionamiento} operante de E. Thorndike y B. F.Skinner

El ejemplo típico de condicionamiento operante nos lo da un jugador que pasa su tiempo frente a una máquina tragamonedas moviendo una palanca repetidamente y esperando que los dibujos aparezcan en una secuencia determinada, cuando ocurre esto, sale de la máquina una lluvia de monedas. En este tipo de aprendizaje, un individuo da una respuesta accidental que es recompensada (reforzamiento), por lo cual el individuo la repite. Si la respuesta no es recompensada lo más probable es que se extin- 
ga. Si se emplea castigo en vez de recompensa las respuestas se anulan.

Edward Lee Thorndike fue el autor de las leyes básicas del condicionamiento operante, sus experimentos consistían en encerrar gatos hambrientos en cajas cerradas y el gato tenía que buscar la forma de salir de la caja para conseguir la comida. Los animales aprendieron, por error y ensayo, a tirar un trozo de cuerda que abría la caja y el truco no se les olvidó permitiéndoles en nuevas experiencias salir de la caja rápidamente. La asociación que hicieron los gatos entre tirar de la cuerda y comer la denominó la ley del "efecto», la cual manifiesta que cuando las acciones de un animal, en cualquier situación dada, son acompanfadas o seguidas por una experiencia satisfactoria, el animal relacionará tal ejecución con la satisfacción obtenida y estará más propenso a repetir las acciones si se encuentra en situaciones simiiares. Burrhus Frederic Skinner es partidario de esta teoría y sli interés fue presentar la manera como un comportamiento afecta el ambiente para producir alguna consecuencia, si esta consecuencia es favorable (refuerzo) la probabilidad de que el comportamiento se repita es alta. Los refuerzos pueden ser positivos o negativos e incrementan la probabilidad de que ocurra o no una conducta. Los refuerzos también pueden ser primarios o secundarios. Los primarios satisfacen necesidades vitales: hambre, sed, etc. y los secundarios son aprendidos, Ilegan a convertirse en refuerzos a tra- vés de su asociación con refuerzos primarios. Existen tres formas de administrar refuerzos: en forma continua, en forma intermitente o por intervalos de tiempo. (Papalia, 1987).

\section{Teoría del aprendizaje Latente de Blodgett}

Las dos teorías anteriores sustentan que si conocemos «las contingencias del refoizamiento podemos explicar y predecir la conducta» del animal o persona, es decir que le están negando toda posibilidad al ser vivo a participar en la elección de su comportamiento, lo cual ha invalidado las teorías principalmente cuando de humanos hablamos. Los psicólogos neo-conductistas aceptan que el medio ambiente juega un papel importante en la construcción de las actitudes de las personas pero dan crédito a que todo ser vivo, ei hombre en mayor grado, desarrolla su propia capacidad para comprender su entorno y tomar iniciativas con relación a lo que debe o no hacer. H.C. Blodgett en 1929 realizó un experimento con ratas para concluir que un individuo aprende de sus experiencias, pero su aprendizaje no lo demuestra mientras no encuentre una motivación para hacerlo. Edward Tolman recogió las experiencias de Blodgett y estudió las reacciones estímulo-respuesta en el aprendizaje en las ratas, concluyendo que entre el estímulo y la respuesta se desarrolla un proceso en el cerebro que le permite aceptar o rechazar estímulos. A raíz 
de sus investigaciones, Tolman cree que el comportamiento obedece más a la comprensión que el individuo desarrolla respecto a la conveniencia o no, de una actuación (orientación hacia una meta), que a un condicionamiento dado (orientación hacia una respuesta). Para Tolman los procesos cerebrales que ocurren entre el estímulo y la respuesta van construyendo en el sujeto un "mapa cognitivo», el cual le permitirá seleccionar una respuesta. Los «mapas cognitivos» no son estáticos, ellos se van actualizando con la información que se obtiene tanto de los sentidos como de las experiencias diarias. (Morgan, 1991).

\section{La teoría del aprendizaje social.}

Albert Bandura ha desarrollado esta teoría, la cual se fundamenta en la idea de que los seres humanos imitamos conductas y comportamientos, que ocurren en situaciones espacificas, de ciertas personas que por algún motivo admiramos. Observando o mirando a otras personas, o escuchando las experiencias vividas por otros, nosotros aprendemos conductas y comportamientos, los cuales serán repetidos mientras produzcan efectos positivos o satisfagan las necesidades. Bandura identificó cuatro pasos en el proceso del aprendizaje social: 1) percibir el comportamiento del modelo en una situación específica, 2) recordar el comportamiento del modelo cuando se presente una situación similar, 3) estar motivado para actuar igual al recuerdo y 4) convertir en acción el recuerdo. (Papalia, 1987).

\section{AMANERA DE SINTESIS}

En el paradigma administrativo aún no existe una teoría lo suficientemente consistente que nos indique cuál es la mejor forma de dirigir a los trabajadores, para que cumpliendo con los objetivos organizacionales que se les asigne estén satisfechos con lo que hacen. En la búsqueda de solución al problema, los psicólogos han elaborado varias teorías que tratan de explicar la conducta de las personas dentro de las organizaciones. Todas estas teorías tienen el problema de que son dificiles de validar, por lo que su generalización es parcial. Las actitudes y la conducta de una persona, de acuerdo a los paradigmas que revisamos anteriormente, parece que dependen de los siguientes factores: 1) de las características biológicas y genéticas heredadas por cada ser, 2) de los patrones culturales aprehendidos durante la infancia, lo cual incluye las satisfacciones y frustraciones que nos hicieron vivir las personas que nos criaron, 3) de los valores morales de una clase socioeconómica que interiorizamos en nuestros inconscientes y que pertenecen al grupo social a donde pertenecemos y 4) de la percepción, definición y estructuración de la situación real y actual a la que debemos responder. Cada uno de estos factores puede ser categorizado dentro de un número muy grande de posibilidades, ahora, 
la combinación de estos cuatro factores nos dará un número infinito de actitudes y conductas, que sustentan ia individualidad de comportamiento de los seres humanos. A pesar de la premisa anterior, se ha encontrado que los seres humanos desarrollamos patrones de conducta bastante similares cuando nos enfrentamos a tipos de situaciones sociales parecidas, esto probablemente a consecuencia del proceso de culturización universal que la sociedad viene sufriendo debido a la popularización de los medios de comunicación, los cuales nos enseñan modos de comportamiento estándares cuando nos toca desempeñar un tipo de rol, ya sea dentro de la familia, con el grupo de amigos o dentro de la organización. Dentro de una empresa se establece un clima organizacional particular en el cual las situaciones sociales nuevas no son lo común, de allí que las actitudes y comportamientos formales de los empleados en su mayoría se «regularizan». Pero aun así, ninguna de las teorías que existen, nos pueden predecir con exactitud el comportamiento de un empleado, lo que nos lleva a que a que el nivel de generalización es realmente bajo en el campo de la conducta humana, ya que son muy complejas las relaciones entre las creencias que tenemos de una situación y las necesidades que deseamos satisfacer en el momento en que se desarrolla la situación. La conclusión a la que podemos llegar es que para lograr entender el funcionamiento de las organizaciones es importante pri- mero llegar a conocer como funcionan los empieados que viven en ella, esto permitirá orientar los estilos de durección que las gerencias deben adoptar para que un trabajador cumpla con los objetivos organizacionales que se le asignan y de otro lado sea un individuo satisfecho con su proyecto de vida.

\section{BIBLIOGRAFIA}

Blank Bubis León. «La administración de organizaciones. Un enfoque estratégico». Cali. Fondo Editorial Universidad del Valle, 1990.

Chiavenato Idalberto. «Introducción a la teoría general de la administración». Editorial Mc Graw-Hill, 1981.

Davila Carlos. "Teorías Organizacionales y administración", Editorial Interamericana, Bogota, 1985.

Davis Keith y Newstrom John. "El comportamiento humano en el trabajo. Comportamiento Organizacional". Editorial. McGraw-Hill, 1988.

Gibson James, Ivancevich John, Donnelly James Jr. "Organizaciones. Conducta, estructura y proceso". Editorial Interamericana, México, 1984.

Hall Richard H. "Organizaciones, estructura y proceso". Madrid. Editorial Prentice Hall, 1983

Kast Fremont E. y Rosenzweig James E. "Administración en las organizaciones. Enfoque de sistemas y contingencias". México. Editorial McGraw Hill, 1987. 
Morgan Gareth. "Imágenes de la Organización"., Editorial Alfaomega, México 1991

Monroy Leonel. "La estructura del ser humano como fundamento de la educación en lo superior y para lo superior". Editorial Univalle, 1994.

Papalia Diane E. / Wendkos Sally. "Sicología". Editorial McGraw-Hill, México, 1987.

Papali Diane / Wendkos Sally, Sicología del desarrollo, Editorial. Mc Graw-Hill, 1985.

Robbins Stephen P, Organizational Behavior, Englewood Cliffs, New Jersey, Edit. Prentice Hall, 1991

Schein Edgar H. "Sicología de la Orga- nización". Madrid, Editorial Prentice Hall International, 1982

Toro Álvarez Fernando Desempeño y productividad. Contribuciones de la sicología Ocupacional, Editorial CINCEL LTDA, Medellín, 1990.

Toro Álvarez Fernando / Cabrera H., Motivación para el trabajo. Conceptos. Hechos y evidencias contemporáneos. Centro de Investigación e Interventoría en comportamiento organizacional. Medellín. 1985.

Williams Clifton y Calas Martha. "Conducta Organizacional". Editorial Scott, Foresman, Glenview Il, 1984. 


\section{Las transformaciones de la prospectiva territorial y la formación de los futuristas: etapas, perfiles, desafíos}

Javier Medina Vásquez.

\section{RESUMIEN}

Los estudios del futuro tienen aplicaciones y énfasis diversos que se han ido diferenciando con el tiempo. En este contexto cabe preguntarse ¿Qué es la prospectıva territorial? ¿Cómo se forma un futurista o prospecivista territoriai?. En el presente artículo se pretende reflexionar sobre esta formación especifica y observar como se han ido transformando los temas dominantes, métodos y prácticas en función de los distintos momentos históricos. $\mathrm{Ai}$ efecto, en la primera parte se hace un breve recuento de la historia de la prospectiva territoriai francesa. Luego se hace énfasis en los apo:tes recientes de la prospectiva de iercera generación en Francia o prospectiva operativa. Después se avanza en los diferentes perfiles, procesos y cortenidos que influyen en la formación específica de los prospectivistas territoriales. Finalmente, aada la tradiciona'. influencia de las prácticas francesas en nuestro continente, se analizan algunos desafíos para la formación de ios futuristas latinoamericanos, conforme a nuestro actual contexto cultural y político-institucional.

Palabras claves: futuro, prospectiva, territorios, formación profesional, Francia, América Latina

\section{ABSTRACT}

The studies of the future have diverse applications and emphases that have been been different with time. In this context it is possible to ask itself What is the territorial prospective? How a territorial one forms futurist or prospectivista. In the present article it is tried to reflect on this specific formation and to observe since they have been become the dominant subjecis, methods and practices based or the different historical moments. To the effect, in the first part a brief count becomes of the history of the French territorial prospective. Soon emphasis in the recent contributions of the prospectiva of third generation in France or operative prospective is made. Later one advances in the different profiles, processes and contents that influence in the specific formation of the territorial prospective experts. Finally, given the traditional influence of the French practices in our continent, I analyze some challenges for the formation of futurist the Latin Americans are analyzed, according to our present cultural and political-institutional context.

Key words: future, prospective, territories, Professional formation, France, Latin America 


\section{¿Y si el Desarrollo fuese una emergencia sistémica?}

\section{Sergio Boisier}

\section{RESUMEN}

El autor plantea una hipótesis audaz que podría tener considerables impactos tanto en el pensamiento teórico como en la práctica del desarrollo territorial. Sostiene el autor que el desarrollo territorial debe entenderse como una propiedad emergente, como una emergencia sistémica de un sistema territorial complejo y con elevada sinergia. Esta es una propuesta completamente opuesta a la práctica corriente de políticas y planes de fomento del desarrollo a escala subnacional, implícitamente basada todavía en un enfoque tipo "incrementalismo disjunto" de Lindblom. Si la hipótesis pasa los filtros metodológicos usuales, habría que re-entrenar radicalmente a los responsables de estos procesos, familiarizándolos con la teoría de sistemas, con la sinapsis neuronal, con la sinergía, con la lógica difusa, con la irreversibilidad temporal, con el caos, etc., con todo lo que aparece detrás de las propiedades emergentes, y habría que reformular también en forma radical la estructura burocrática y el funcionamiento de los cuerpos políticos y técnicos que se desempeñan en el terreno. El desarrollo hay que re-escribirlo en el marco del paradigma de la complejidad, y en un marco humanista y constructivista como el usado por el autor.

Palabras claves: sistemas, propiedades emergentes, sinapsis, sinergía, desarrollo territorial, complejidad.

\section{ABSTRACT}

The author iaises an audacious hypothesis with considerable impacts in the theoretical thought as actually of the territorial development. He maintains that teiritorial development must be understood like an emergent property, like a systemic emergency of a complex territorial system and with elevated sinergia. This is a proposal completely opposed to the current practice of policies and plans of promotion of the subnational scale development, implicitly based still the approach "separated incrementalism" type of Lindblom. If the hypothesis passes methodologic the usual filters, would be necessary re-to train radically to the people in charge of these processes, familiarizing them with the theory of systems, the neuronal synapse, sinergia, the fuzzy logic, the temporary irreversibilidad, the chaos, etc. Ye! what it appears behind the emergent properties, and would be necessary also reformulate in radical form the bureaucratic siructure and the operation of the political and technical bodies that evolve in the land. The development is necessary within the framework to rewrite it of the paradigm of the complexity, and in a frame of constructivism humanist view and like the used one by the author.

Key words: emergent systems, properties, synapse, sinergía, territorial development, complexity. 


\section{La construcción de Región como ejercicio en la complejidad}

\section{RESUMEN}

El presente ensayo aborda el tema de construcción de región, un proceso relativamente reciente en Colombia. con dos especificidades. La primera consiste en que el enfoque es de orden epistemo:ógico: se plantea la tesis de que la construcción de región exige un cambio fundamental en los supuestos cognitivos tradicionales. La segunda especificidad es que la reflexión tiene como referente práctico el proceso de constricción de región que se viene adelantando en seis departamentos del sur de Colombia (Caquetá, Cauca, Huila, Nariño, Putumayo y Tolima), en lo que se ha denominado "Iniciativa Surcolombiana". Se trata, entonces, de un atrevido intento por conectar dos puntos extremos: la disquisición más abstracta (condiciones del pensamiento) con un proceso de construcción social. Este intento se traduce en dos hipótesis básicas que ponemos a consideración, para validación o negación, de quienes estamos pensando y actuando por la construcción de regiones en Colombia: lâ primera, que al proceso, para avanzar más rápidamente, le hacen falta dosis importantes de pensamiento complejo difundido ampliamente. La segunda que, además de elementos básicos de complejidad en la forma de pensar los asuntos, se requiere una práctica co-

\section{Rodrigo Quintero}

rrespondiente con esa complejidad de los conceptos. Es decir que, con frecuencia, en la práctica, en las propuestas concretas, en las realizaciones, retrocedemos (por facilidad, incapacidad o temor ante la integralidad y complejidad de la vida social) hacia metodologías simplificantes, reduccionistas, incongruentes con la complejidad de las concepciones.

Palabras claves: región, complejidad, Colombia, cambio social, construcción socia!

\section{ABSTRACT}

This article approaches the subject of construction of region, a relatively recent process in Colombia, with two specificities. First it consists of which the approach is of epistemologic order: the thesis considers of which the construction of region demands a fundamental change in the traditional cognitives assumptions. The second, specificity is that the reflection has like referring practitioner the process of construction of region that comes advancing in six departments from the south of Colombia (Caquetá, the Cauca, Huila, Nariño, Puizmoryo and Tolima), in which has denominated "Iniciativa Surcolombiana". One treats, then, on a bold attempt to connect two end points the most abstract 
disquisition (conditions of the thought) with a process of social construction. This attempt is translated in two basic hypotheses that we put to consideration, for validation or negation, of those who we are thinking and acting by the construction of regions about Colombia: first, that to the process, to advance more quickly, widely needs important doses to him of spread complex thought. Second that, in addition to basic elements of complexity in the form to think the subjects, requires a corresponding prac- tice with that complexity of the concepts. It is to say that, frequently, ac. tually, in the concrete proposals, the accomplishments, we back down (by facility, incapacity or fear before the integrality and complexity of the social life) towards simplicity and redutionism view, whit incongruous methodologies of the conception complexities.

Key words: region, complexity, Colonbia, social change, social cons. truction 


\title{
Modernidad, sentimientos negativos y conflicto social en Colombia
}

\author{
Fernando Cruz Kronfly
}

\section{RESUMEN}

En este artículo pretendo demostrar cómo, además de los factores económicos, políticos y sociales tradicionalmente identificados como capaces de originar y exacerbar el conflicto social en el mundo moderno, existen otros generalmente no evidentes ni mucho menos reconocidos por la teoría en su importancia, salvo significativas excepciones, por su carácter inconsciente. Se trata de los denominados «sentimientos negativos» que se asocian a la conducta humana, tales como la envidia, la ambición, el odio, la sed de venganza y de reparación del daño sufrido, así como el resentimiento, entre otros, que en el curso de la historia de ciertos pueblos y en determinadas circunstancias, han terminado apoderándose de la dinámica social, descentrando el conflicto de sus fines «nobles» y ejes principales.

Palabras clave: Colombia, modernidad, sentimientos negativos, conflicto social, inconsciencia

\section{ABSTRACT}

In this article I try to demonstrate how, in addition to economic, political and social the factors traditionally identifled like able to originate and to increase the social conflict in the modern world, nonevident ones exist generally other far from it recognized by the theory in their importance, except for significant exceptions, by their unconscious character. One is the denominated "negative feelings" that are associated to the human conduct, such as it envies it, the ambition, hatred, the thirst of revenge and repair of the suffered damage, as well as the resentment, among others, that in the course of the history of certain towns and in certain circumstances, have ended up seizing of social dynamics, putting off center the conflict of their "noble" aims and main axes.

Key words: Colombia, negative modernity, feelings, social conflict, unconsciousness 


\title{
El Comportamiento Humano
}

\author{
Carlos Eduardo Cobo
}

\section{RESUMEN}

Hace un año aproximadamente me interese en estudiar las causas del comportamiento humano, ya que soy un convencido de que la fuerza básica que mueve cualquier empresa nace del ser mismo cuando se aan ciertas condiciones intrínsecas y extrínsecas a él. El problema del comportamiento humano ha tenido diferentes soluciones a través de la historia, desde antes de que se iniciaran las investigaciones psicológicas, filósofos, antropólogos y teólogos desarrollaron constructos acerca del porque del comportamiento de una persona en una determinada situación. Este articulo pretende hacer una síntesis incompleta de las diferentes teorías o paradigmas que han intentado resolver el problema. ile agrupado las Teorías que explican las actitudes y los comportamientos humanos en 3 categorías buscando facilitar al lector la comprensión de los elementos comunes y diferentes entre los modelos

Palabras claves: comportamiento humano, investigación social, empresarismo, ciencias sociales

\section{ABSTRACT}

I'm interesting since for a year, approximately, in studying the causes of the human behovior, since I am convincing that the basic force that moves any company is born of the same being when certain intrinsic and extrinsic conditions occur him. The problem of the human behavior has had different solutions through history, from before the psychological investigations began, philosophers, anthropologists and theologians developed ideas about because this subject centering of the behavior of a person in a certain situation. I tries to make a synthesis incomplete of the different theories or paradigms that have tried to solve the problem. I have grouped the Theories that explain the attitudes and the human behoviors in 3 categories, looking for to facilitate to the reader ine understanding of the common and different elements between the models

Key words: human behovior, social investigation, entrepreneurship, social sciences 


\section{Criterios de análisis para estudiar los aspectos sectoriales del entorno económico para las microempresas de Ingeniería de Proyectos, Sector Hidráulico en Santiago de Cali \\ Carlos Tello Castrillón}

\section{RESUMEN}

En el presente escrito se tratan las premisas básicas que guían el estudio de los aspectos sectoriales del entorno económico, aplicándolos al caso especifico de las microempresas de Ingeniería de proyectos, sector hidráulico. La discusión empieza por definir el sector de estudio y su umportancia. Luego aborda lo que se entiende por entorno y dentro de este cuales son los aspectos sectoriales. Para el objeto de estudio escogido se presentan algunas dificultades sobre el marco teórico del tema, puesto que son claras algunas ambigüedades e indefiniciones que obligan a una acotación de los términos. Al final, incluyendo un pequerio trabajo de campo en el que se sumaron entrevistas y relación de experiencias, se hace la caracterización inicialmente propuesta, que constituye una aproximación al estudio estratégico de las microempresas referidas.

\section{ABSTRACT:}

In the present written treat the basic premises that guide the study of the sectorial aspects of the economic environment, applying therri al specific case of the projects Engineering micro-businesses, hydraulic sector. The discussion begins for defining the sector of study and its importance. Then it undertakes what is understood for environment and inside this which are the sectorial aspects. For the object of study chosen some difficulties on the theoretical framework of the theme are presented, since are clear some ambiguities and lacks of definition that oblige an acotacion of the terms. Al final, including a small work of field in which they added interviews and relation of experiences, the intially proposed characterization is done, that constitutes an approximation al strategic study of the micro-busines ses referred. 